\title{
Observational tests of planet formation models
}

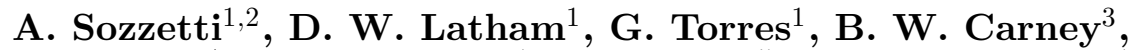 \\ J. B. Laird ${ }^{4}$, R. P. Stefanik ${ }^{1}$, A. P. Boss $^{5}$, D. Charbonneau ${ }^{1}$, \\ F. T. ODonovan ${ }^{6}$, M. J. Holman ${ }^{1}$ and J. N. Winn ${ }^{7}$ \\ ${ }^{1}$ Harvard-Smithsonian Center for Astrophysics, Cambridge, MA 02138, USA \\ email: asozzett@cfa.harvard.edu \\ ${ }^{2}$ INAF- Astronomical Observatory of Torino, I-10025 Pino Torinese (TO), Italy \\ ${ }^{3}$ University of North Carolina at Chapel Hill, Chapel Hill, NC 27599, USA \\ ${ }^{4}$ Bowling Green State University, Bowling Green, OH 43403, USA \\ ${ }^{5}$ Carnegie Institution of Washington, Washington DC 20015, USA \\ ${ }^{6}$ California Institute of Technology, Pasadena, CA 91125, USA \\ ${ }^{7}$ Massachusetts Institute of Technology, Cambridge, MA 02139, USA
}

\begin{abstract}
We summarize the results of two experiments to address important issues related to the correlation between planet frequencies and properties and the metallicity of the hosts. Our results can usefully inform formation, structural, and evolutionary models of gas giant planets.
\end{abstract}

\section{Introduction}

The planet-metallicity connection is one of the most important aspects of the close relationship between characteristics and frequencies of planetary systems and the physical properties of the host stars which have been unveiled by the present sample of over 200 extrasolar planets. In particular, the likelihood of finding a planet around a given star rises sharply with stellar metallicity (e.g., Santos et al. 2004; Fischer \& Valenti 2005). Furthermore, a correlation may also exist between estimated inner core masses of transiting giant planets and the hosts' metal content (Guillot et al. 2006; Burrows et al. 2007). In both cases, the evidence collected so far appears to strongly support the more widely accepted mechanism of giant planet formation by core accretion (e.g., Pollack et al. 1996), as opposed to the alternative formation mode by disk instability (e.g., Boss 1997). However, the relatively small numbers of metal-poor stars screened for planets so far, and the large uncertainties often present in the determination of both planet and stellar properties in transiting systems prevent one from drawing conclusions. We describe two experiments designed to put the observed trends on firmer observational grounds, thus ultimately helping to discriminate between proposed planet formation models. The first is a Doppler survey for giant planets orbiting within $2 \mathrm{AU}$ of a well-defined sample of 200 field metal-poor dwarfs. Our data can help to gauge the behavior of planet frequency in the metal-poor regime. The second consists of a novel method for improving on the knowledge of stellar and planetary parameters of transiting systems through a careful analysis of spectro-photometric measurements. With this approach, structural and evolutionary models of irradiated planets can be better informed, allowing for refined estimates of the heavy-element content of transiting planets and for improved understanding of the core mass - stellar metallicity correlation. 


\section{Testing the $f_{p^{-}}[\mathrm{Fe} / \mathbf{H}]$ correlation}

Based on an analysis of over 3 yr of precision radial velocity measurements of $\sim 200$ metal-poor stars observed with HIRES on the Keck 1 telescope (Sozzetti et al. 2006), we have identified several long-term, low-amplitude radial-velocity variables, which we are following up with direct imaging techniques at infrared wavelengths. We have placed upper limits on the detectable companion mass as a function of orbital period. Our survey would have detected, with a 99.9 confidence level, over $95 \%$ of all companions on low-eccentricity orbits with velocity semi-amplitude $K \gtrsim 100 \mathrm{~m} \mathrm{~s}^{-1}$, or $M_{p} \sin i \gtrsim$ $4.1 M_{\mathrm{J}}(P / y r)^{(1 / 3)}$, for orbital periods $P \lesssim 3 \mathrm{yr}$. None of the stars in our sample exhibits radial-velocity variations compatible with the presence of Jovian planets with periods shorter than the survey duration. The resulting average frequency of gas giants orbiting metal-poor dwarfs with $-2.0 \lesssim[\mathrm{Fe} / \mathrm{H}] \lesssim-0.6$ is $f_{p}<0.67 \%$. We examine the implications of this null result in the context of the observed correlation between the rate of occurrence of giant planets and the metallicity of their main-sequence solar-type stellar hosts. By combining our dataset with the Fischer \& Valenti (2005) uniform sample, we confirm that the likelihood of a star to harbor a planet more massive than Jupiter within 2 AU can be expressed as a quadratic function of the host's metallicity. However, the data for stars with $-1.0 \lesssim[\mathrm{Fe} / \mathrm{H}] \lesssim 0.0$ are compatible, in a statistical sense, with a constant occurrence rate $f_{p} \simeq 1 \%$ (see also Santos et al. 2004).

\section{Testing the $M_{c^{-}}[\mathrm{Fe} / \mathrm{H}]$ correlation}

Using high-resolution Keck spectra, we derive improved atmospheric parameters for the parent stars of the recently discovered transiting planets TrES-3 (O'Donovan et al. 2006) and TrES-4 (Mandushev et al. 2007). TrES-3 is a mildly metal-deficient G-dwarf with $[\mathrm{Fe} / \mathrm{H}]=-0.19 \pm 0.08$, while TrES-4 is a slightly evolved, mildly metal-rich Fstar with $[\mathrm{Fe} / \mathrm{H}]=0.14 \pm 0.09$. We determine stellar masses and radii using the combined spectro-photometric approach described in Sozzetti et al. (2007), in which $T_{\text {eff }}$ and the normalized separation $a / R_{\star}$ are used in the comparison with stellar evolution models. We obtain $M_{\star}=0.924_{-0.040}^{+0.012} M_{\odot}, R_{\star}=0.813_{-0.027}^{+0.012} R_{\odot}$ for TrES-3 and $M_{\star}=1.384_{-0.046}+0.070 M_{\odot}, R_{\star}=1.810_{-0.056}^{+0.071} R_{\odot}$ for TrES-4. The improved planetary radius thus inferred confirms TrES- 4 as the planet with the largest radius (and lowest density) discovered so far. Given the super-solar metallicity of its host, the TrES-4 system (together with WASP-1 and HAT-P-4) suggests that gas giant planet core mass is not a simple function of host-star metallicity or of radiation environment. We conclude that more definite statements on the relation of the observations and planet structure theories can be made only by reaching higher accuracy in the observed star/planet parameters.

\section{References}

Boss, A. P. $2000 A p J$ (Letters), 536, L101

Burrows, A., Hubeny, I., Budaj, J., \& Hubbard, W. B. 2007, ApJ, 661, 502

Fischer, D. A. \& Valenti, J. 2005, ApJ, 622, 1102

Guillot, T., Santos, N. C., Pont, F., Iro, N., Melo, C., \& Ribas, I. 2006, A\&A (Letters), 453, L21

Mandushev, G., et al. 2007, ApJ (Letters), 667, L195

O'Donovan, F. T., et al. 2007, ApJ (Letters), 663, L37

Pollack, J. B., et al. 1996, Icarus, 124, 62

Santos, N. C., Israelian, G., \& Mayor, M. 2004, A\& A, 415, 1153

Sozzetti, A., et al. 2006, ApJ, 649, 428

Sozzetti, A., et al. 2007, ApJ, 664, 1190 